\title{
Instituciones, organizaciones y democracia: una revisión de los enfoques teóricos para el análisis de la gestión estatal en América Latina
}

Institutions, organizations and democracy: a review of theoretical approaches for the analysis of state management in Latin America

Instituições, organizações e democracia: uma revisão dos enfoques teóricos para a análise da gestão estatal na América Latina

\author{
Pablo Andrade Andrade \\ Universidad Andina Simón Bolívar, Sede Ecuador \\ pablo.andrade@uasb.edu.ec
}

DOI: https://doi.org/10.32719/25506641.2020.7.4

Recibido: 15 de julio de 2019 • Revisado: 20 de octubre de 2019

Aceptado: 5 de noviembre de 2019

Artículo de investigación

Licencia Creative Commons

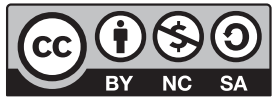




\section{Resumen}

El artículo hace una revisión teórica de la evolución del problema de las instituciones democráticas en las democracias recientes, con el objetivo de presentar al lector los enfoques estándar para estudiar la relación entre régimen político y política pública. Se argumenta que los académicos han dejado de tratar a la democracia desde una perspectiva centrada en los componentes del régimen político, para enfocarse en el funcionamiento de las instituciones políticas con relación a la gestión estatal. El ensayo examina tres vertientes de esa nueva ola de investigaciones: la economía política mainstream, el enfoque de las instituciones informales y la perspectiva de los acuerdos políticos. El examen pone particular atención al lugar que los tres enfoques otorgan a las organizaciones como actores políticos. El autor concluye presentando las implicaciones de esa evolución teórica para una agenda de investigación empírica que podría desarrollarse a partir de la confluencia entre la economía política y los estudios organizacionales.

Palabras clave: Instituciones, políticas, organizaciones, teorías, cambio.

JEL: L38 Política pública.

\section{Abstract}

The article makes a theoretical review of the evolution of the problem of democratic institutions in recent democracies, presenting the standard approaches to study the relationship between political regime and public policy. It is argued that academics have stopped treating democracy from a perspective focused on the components of the political regime to concentrate on the functioning of political institutions in relation to state management. The essay examines three aspects of this new wave of research: mainstream political economy, the approach of informal institutions, and the perspective of political agreements. The review pays particular attention to the place that these three approaches give organizations as political actors. The author concludes by presenting the implications of this theoretical evolution for an empirical research agenda that can be developed from the confluence between political economy and organizational studies.

Keywords: Institutions, policies, organizations, theory, change.

JEL: L38 Public policy.

\section{Resumo}

O artigo faz uma revisão teórica da evolução do problema das instituições democráticas nas democracias recentes, com o objetivo de apresentar ao leitor os enfoques padrões para estudar a relação entre regime político e política pública. Argumenta-se que os acadêmicos deixaram de tratar a a democracia desde uma perspectiva centrada nos componentes do regime político para se enfocarem no funcionamento das instituições políticas com relação à gestão estatal. $\mathrm{O}$ ensaio analisa três vertentes dessa nova onda de pesquisas: a economia política mainstream, o enfoque das instituições informais e a perspectiva dos acordos políticos. A análise dá uma atenção especial ao lugar que os três enfoques outorgam às organizações como atores políticos. $\mathrm{O}$ autor conclui apresentando 
as implicações dessa evolução teórica para uma agenda de pesquisa empírica que pode se desenvolver a partir da confluência entre a economia política e os estudos organizacionais.

Palavras-chave: Instituições, políticas, organizações, teoria, mudança.

JEL: L38 Políticas Públicas.

\section{Introducción}

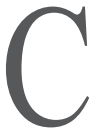

uando empezó la tercera ola de democratización en América Latina hace 40 años, ${ }^{1}$ el debate sobre la región, en ciencia política, estuvo dominado por dos condiciones. En primer lugar, la urgencia del momento validaba a la teoría de las transiciones. En efecto, esta óptica articulaba de manera elegante las preocupaciones por la creación de condiciones que hicieran posible el paso de los regímenes autoritarios que existían en la región hacia nuevos regímenes caracterizados por la competencia electoral, la reinstalación de las libertades liberales clásicas, la institucionalización de varias formas de control al poder de los Ejecutivos sobre los Estados, y más generalmente del poder de estos últimos sobre los ciudadanos. La segunda condición fue el desarrollo todavía muy incipiente de la ciencia política en América Latina, y en particular del estudio desde la óptica de las políticas públicas. La combinación de las dos condiciones determinaría la evolución de los debates latinoamericanos sobre las instituciones políticas.

La influencia de la teoría de las transiciones se prolongó hasta la actualidad, pero no sin cambios. A partir de la década de los 90, cuando el ciclo de las transiciones se cerró en la mayor parte de la región, ${ }^{2}$ el eje de la indagación pasó a centrarse - aunque no exclusivamente- en la calidad de la democracia, y los problemas latinoamericanos de antigua data (v. g., crecimiento económico sostenido sin distribución y múltiples desigualdades persistentes) se entendieron como resultado de la deficiente calidad de las instituciones de-

1. Ecuador fue el primer país latinoamericano de esa ola, con las elecciones de 1978 y la inauguración del primer gobierno democrático en agosto de 1979.

2. Chile y Paraguay finalizaron sus transiciones en 1989. México, gracias a la combinación entre competencia electoral y partido dominante, fue un caso problemático y relativamente marginado para la teoría de las transiciones, hasta la primera derrota electoral del PRI en 2000 (Greene 2009). 
mocráticas. Por su parte, en Latinoamérica la ciencia política no ha logrado todavía instalarse como una disciplina fuerte y en plena expansión (Altman 2005 y 2012), de manera que, en el campo de la investigación sobre las instituciones políticas latinoamericanas, la reflexión regional no ha logrado crear marcos teóricos enfocados ${ }^{3}$ que permitan poner en duda las prescripciones de las agencias internacionales para el diseño y la implementación de políticas públicas. En síntesis, el problema ha dejado de ser la democratización, pero la urgencia persiste en la tarea de "mejorar las instituciones políticas".

El presente trabajo no sigue el camino usual de los debates latinoamericanos, no busca ofrecer respuestas metodológicas o soluciones a cómo intervenir políticamente. El propósito del ensayo es ofrecer al lector alternativas teóricas para conceptualizar la política de las políticas públicas y su relación con el funcionamiento de las democracias latinoamericanas. El trabajo revisa dos cuerpos de literatura académica que se han propuesto como marcos teóricos enfocados para la investigación -y el mejoramiento- de las instituciones políticas y las agencias estatales. El primer conjunto teórico se ocupa del problema de la fortaleza de las instituciones formales. La segunda perspectiva es conocida en el mundo anglosajón como el enfoque de los "acuerdos o fundamentos políticos". ${ }^{4}$ A pesar de sus diferencias, esas orientaciones comparten su preocupación por cómo funcionan las reglas formales de juego del régimen político en interacción con constelaciones de poder que pueden estar o no formalmente codificadas. Como se verá a continuación, las perspectivas se originan en el resurgimiento de la economía política como campo de investigación social. Las teorías, sin embargo, difieren en cuanto a la forma de pensar los cambios político-institucionales que cabe esperar en las sociedades latinoamericanas; es precisamente en este aspecto predictivo que investigaciones futuras podrían ponerlas a prueba.

3. Rueschmeyer (2009) propone el término para designar a conjuntos conceptuales y metodológicos que inspiran hipótesis para la investigación localizada que pueden ponerse a prueba empíricamente, de manera que se identifiquen las fronteras de su aplicabilidad y se incorporen estos resultados en marcos teóricos revisados.

4. Traduzco la expresión original inglesa political settlements como 'acuerdos' o 'fundamentos', aunque, como se expone más adelante, tal vez sería más apropiado hablar de asentamientos o sedimentaciones políticas. 


\section{¿Es el régimen o son las instituciones políticas?}

Tras una exhaustiva revisión de cuatro influyentes trabajos publicados a inicios del siglo XXI sobre los procesos de democratización de la Europa del siglo XIX, Daniel Zibblat (2006) sugiere un cambio en la agenda de investigación comparativa sobre los regímenes modernos. Las teorías sobre la democratización se han concentrado en la explicación de dos tipos de resultados políticos, los autoritarismos y las democracias, en un cierto período temporal (por ejemplo, la América Latina de los años 60 a los 80, o de las dos últimas décadas del siglo XX), lo cual ha contribuido a crear narraciones sobre procesos graduales de transición de uno a otro tipo de régimen y al uso de criterios de umbral que marcan el paso de uno a otro tipo polar. ${ }^{5}$

Sin embargo, es teóricamente posible dividir esa trayectoria gradual en varios episodios de introducción y cumplimiento de instituciones democráticas que coexisten con instituciones no democráticas. Zibblat (2006) indica que este cambio en "la unidad de análisis", del régimen como totalidad institucional a las reformas democratizantes, abre una rica agenda de investigación, cuyo examen podría ayudarnos a identificar distintas configuraciones institucionales y sus respectivas coaliciones de apoyo dentro de un mismo tipo general de régimen, distintas combinaciones y secuencias de democratización, así como las posibles consecuencias de esos patrones para la instalación del paquete institucional completo que conocemos como régimen democrático.

La propuesta de Zibblat puede parecer un mero tecnicismo académico, pero no lo es. De hecho, pone en duda la sabiduría recibida a partir de la teoría de las transiciones. En efecto, una comparación entre las distintas trayectorias del gradualismo europeo (y de Estados Unidos, Canadá, Australia y Nueva Zelanda) y las democratizaciones latinoamericanas de la tercera ola resalta la brusquedad del cambio institucional en esta última región. En la década de los 80 , la más o menos precipitada fuga de los militares fuera del control del Estado produjo una rápida instalación del paquete completo del régimen democrático, bajo el supuesto de que sus distintas arenas insti-

5. La democracia moderna estaría dada por la existencia simultánea de tres conjuntos institucionales: el sufragio universal masculino, una legislatura autónoma y las libertades civiles. 
tucionales mantenían entre sí relaciones armónicas de complementariedad. La perspectiva dominante, la teoría de las transiciones, sostenía que la instalación del paquete a lo largo del Estado tomaría tiempo, pero no preveía la posibilidad de que las arenas institucionales del régimen político pudieran tener variadas configuraciones de reglas funcionando según sus propias lógicas conflictivas; unas dirigiéndose hacia la consolidación de instituciones autoritarias y otras, hacia la democracia. ${ }^{6}$

La teoría de las transiciones, en la práctica, llevaba a dejar de lado una importante comprensión que, por un lado, el institucionalismo histórico comparativo y, por el otro, la nueva economía política institucional venían desarrollando en paralelo; a saber, que las secuencias de instalación de un cierto conjunto institucional tienen una importancia decisiva en la interacción con otros conjuntos institucionales. La historia y los ritmos de cambio institucional cuentan para el agregado final (el régimen). La perspectiva tampoco tomaba en cuenta que las instituciones, en tanto reglas de juego que facilitan la coordinación social, emergen de manera distinta en países con una larga -y exitosa- trayectoria de desarrollo y en otros con trayectorias erráticas $-\mathrm{y}$ muchas veces fracasadas-. En síntesis, la expectativa de la no variación de resultados de la instalación de regímenes democráticos en América Latina pecaba de formalismo; asumía que el nuevo paquete institucional (con sus cuidadosos diseños constitucionales, de sistemas electorales, cortes constitucionales, roles estatales en el manejo económico, etc.) podía asentarse sin mayor problema en países cuyas trayectorias históricas diferían significativamente de las de los países capitalistas avanzados.

Empíricamente, la perspectiva tendía a dejar fuera el examen de un reto central para las instituciones políticas de las sociedades latinoamericanas. En efecto, las grandes transformaciones desatadas por los procesos de industrialización del siglo XX aún se encontraban en curso; en particular, la evolución del capitalismo en la región dependía fuertemente de la intervención estatal. ${ }^{7}$ Por lo tanto, el análisis político no podía partir de una demarcación clara

6. La idea de reductos autoritarios no sustituye ese déficit teórico, porque se refiere a la existencia de privilegios y protecciones a las fuerzas armadas o a los antiguos dictadores, no a la interacción entre las arenas institucionales del régimen político.

7. Asunto que fue tomado en cuenta como problema por la nueva sabiduría de la economía del desarrollo de tintes neoliberales (Evans y Stallings 2016). 
entre las instituciones del régimen político y aquellas otras que buscaban gobernar la evolución económica. La puesta en marcha, en la década de los 90, del Consenso de Washington puso en evidencia las consecuencias de ese sesgo empírico. La instalación del neoliberalismo en su forma completa ${ }^{8}$ desató una lucha política intensa por las políticas de desarrollo y sus resultados, y puso en el primer plano de las preocupaciones académicas -y de las instituciones financieras internacionales- la cuestión de la relación entre cambio institucional político y resultados de las políticas públicas para el desarrollo. ${ }^{9}$ Este desplazamiento de problemáticas supuso un cambio de eje del análisis institucional del régimen (del conjunto institucional que gobierna el Estado) a una nueva unidad analítica (las instituciones políticas como variable causal).

A fines de la década anterior, el Banco Interamericano de Desarrollo (BID) publicó un libro que resumía el nuevo énfasis otorgado al análisis institucional de las políticas, Policymaking in Latin America. How Politics Shapes Policies (Stein y Tommasi 2008). Los trabajos contenidos en este influyente documento ${ }^{10}$ no solo abandonaban el análisis del régimen por el de las instituciones, sino que también partían de un supuesto común, sintetizado por Rodrik (2007) en un análisis comparativo de los resultados de desarrollo luego de dos décadas de reformas neoliberales en el mundo: para explicar los resultados de una política de desarrollo, más importante que el contenido de una política son su estabilidad y su aplicación real. La cuestión de las mejores instituciones -con su énfasis en el diseño institucional- quedaba atrás. La nueva agenda de investigación se enfocaba en la indagación de los procesos políticos de decisión que hacen posible o impiden la cooperación entre los

8. El neoliberalismo puede entenderse como una forma específica de capitalismo que reorganiza las relaciones económicas según principios neoclásicos y las relaciones políticas bajo la forma de una democracia liberal; esto es, un Estado con capacidades mínimas para la intervención económica, con gobiernos constituidos por la competencia electoral entre élites políticas (Silva y Rossi 2018; Silva 2009; Andrade 2008).

9. Entendidas como las respuestas (o ausencia de respuestas) de los gobiernos a los conflictos ocasionados por sus iniciativas para obtener un mercado libre de distorsiones estatales y la reestructuración de las instituciones sociales.

10. Jones (2017) afirmaba casi una década después de su publicación que el libro original en inglés y su traducción al español (2010) eran de uso corriente en la academia y una de las fuentes de consulta más frecuentes para analistas, ejecutivos de negocios, diplomáticos, periodistas, decisores políticos, y políticos fuera del Estado. 
actores políticos y sociales que hacen las políticas de desarrollo en un país. En esta perspectiva, el objeto de estudio es la política de las políticas públicas (en un amplio rango que va desde la construcción de burocracias hasta el diseño de estrategias impositivas).

La perspectiva desarrollada por el BID enfatiza el proceso de creación de políticas (en adelante, PCP) como la variable causal de la eficiencia de las políticas adoptadas por un gobierno. En pocas palabras, la investigación del PCP permite explicar por qué ciertas políticas logran obtener los resultados que los gobiernos se proponen y otras no. La teoría se construye a partir de una concepción del PCP como un juego estratégico entre actores formales: si el juego es cooperativo, entonces las políticas serán eficientes; si no lo es, los resultados esperados no se obtendrán.

La teoría concibe a las políticas como resultado de las interacciones (cooperativas -de negociación- o no cooperativas - de imposición-) entre los grupos poderosos de una sociedad nacional (élites gubernamentales y/o estatales, élites de negocios organizadas en asociaciones y/o grupos económicos, líderes de partidos y movimientos políticos, élites de organizaciones no gubernamentales por sector de interés, y dirigentes de varios tipos de organizaciones de los sectores populares). Esas interacciones se encuentran enmarcadas por instituciones políticas formales que proveen (o no) recursos y oportunidades para la cooperación. Esto es, las instituciones políticas formales tienen la capacidad para transformar juegos no cooperativos en cooperación. Ahora bien, la capacidad transformadora de las instituciones políticas formales existe cuando estas comparten al menos dos características: facilitan la interacción continua entre actores, y hacen posible que estos extiendan sus horizontes temporales del corto al mediano y largo plazo (Spiller, Stein y Tommasi 2006). Metodológicamente, el análisis requiere contar con información sobre los actores clave que participan del PCP, los recursos de poder que controlan y los roles que juegan, sus preferencias, incentivos y capacidades, los horizontes temporales de los actores, las arenas en las que interactúan, si estas están o no formalmente reguladas, y, finalmente, la naturaleza de los intercambios/transacciones políticas en que se involucran.

Armados con este aparato analítico es posible, entonces, formular recomendaciones de política dirigidas hacia los actores que participan en el PCP, y orientadas a mejorar las características de las instituciones políticas 
formales que lo enmarcan; esto es, aumentando gradualmente la capacidad de estas instituciones para facilitar la cooperación entre los actores involucrados. Adicionalmente, el método permite desarrollar métricas sobre las características de las instituciones formales y de las políticas públicas adoptadas por un gobierno, y dado que los resultados de estas son también medibles, es posible identificar y explicar las variaciones de resultados de las políticas económicas, ambientales y de incorporación social adoptadas por un gobierno. Esta métrica complementa las medidas estándar de costos de inversión, y por su sensibilidad a la variación hace posible distinguir los grados de eficiencia entre los diferentes sectores de intervención estatal y facilita la comparación entre países.

En definitiva, estamos en un retorno a la economía política en su acepción más clásica, ${ }^{11}$ pero sobre la base de las correcciones introducidas por la nueva economía institucional a la economía neoclásica. El origen de esa fundamentación teórica es, sin embargo, más cercano a la corriente principal de la ciencia política anglosajona que a la economía. En particular, el enfoque de la eficiencia deriva su poder analítico de la síntesis entre el programa original del institucionalismo histórico comparativo y el de la teoría de la acción racional, un desarrollo que ocurrió entre fines de los 90 y la primera década del presente siglo en el campo de la política comparada.

La elegancia del enfoque, así como su claridad metodológica, ha permitido ponerlo a prueba en varias ocasiones. Además de los estudios originales de 2007, hubo réplicas parciales en 2014 y 2017 (Jones 2017). Para Ecuador, el trabajo original de 2007 inspiró una ingeniosa afinación metodológica para la comprensión de la primera década de gobiernos de Alianza País (Polga-Hecimovich y Sánchez 2018). Sin embargo, algunas de las conclusiones empíricas para ciertos casos nacionales han sido puestas en duda (Luna 2014).

El enfoque tiene dos problemas. En primer lugar, la variación empírica entre instituciones políticas (la variable causal que explica la calidad de las políticas) no puede ser explicada dentro del marco teórico del PCP; este simplemente asume que esa variabilidad existe y puede disminuirse mediante las políticas adecuadas, lo cual implica una tautología. Segundo, el enfoque

11. Esto es, el estudio de la sociedad enfocado en los factores políticos y sociales que crean prosperidad o riqueza, a diferencia de la concepción neoclásica de la economía como "ciencia de la escasez" (Mazzucato 2018, 34). 
asume una evolución institucional gradual; esto es, supone que las instituciones en una democracia permanecen estableces por largos períodos, y solo en ciertas coyunturas críticas hay cambios acelerados; el ritmo de cambio esperado sería lento y acumulativo. Este segundo supuesto es particularmente problemático ante la evidencia empírica latinoamericana. Las dos teorías alternativas que examinaré a continuación toman en cuenta los problemas señalados.

\section{Variación institucional formal}

Al desplazar el objeto de investigación de la calidad (eficiencia) de las políticas al de las características de las instituciones formales, el enfoque PCP inadvertidamente asume que esas instituciones escritas en constituciones y otros instrumentos legales ${ }^{12}$ generan expectativas compartidas entre los actores participantes del proceso político, y que estas orientaciones prevalecen por largos períodos de tiempo. Sin embargo, en América Latina (y en gran parte de los países en desarrollo) los actores no buscan estabilizar las reglas de juego sino cambiarlas constantemente. Simplificando, podría decirse que la expectativa es que las instituciones políticas formales actúan durante un cierto tiempo más como una sugerencia que como un dictamen formal de cumplimiento obligatorio, y que pasado ese tiempo son modificadas. En estas condiciones, la indagación por la fortaleza (o debilidad) institucional adquiere relevancia.

Levitsky y Murillo (2009) han propuesto desagregar el concepto de fortaleza institucional en dos dimensiones cuyas variaciones explicarían la variación de las instituciones tanto al interior de un país como entre países. Al entender a las instituciones políticas formales como "reglas y procedimientos que restringen y hacen posible la conducta política" (Levitsky y Murillo $2009,117)$, los autores sostienen que es posible distinguir patrones de fortaleza y debilidad de las instituciones políticas formales que responden a la variación (simultánea o independiente) en relación con la estabilidad y el

12. Carey $(2000,735)$ llama "reglas de pergamino" a todas las instituciones políticas escritas en leyes, reglamentos, constituciones, tratados, etc. 
cumplimiento. ${ }^{13}$ Los autores identifican cuatro tipos de situaciones en las que pueden encontrarse variaciones empíricas de la estabilidad y el cumplimiento, a saber: instituciones altamente estables y de alto cumplimiento; reglas formales que perduran, pero son solo débilmente cumplidas; instituciones a las que se obedece regularmente, pero que son de corta duración; y, finalmente, reglas formales que cambian con facilidad (son inestables) y se hacen cumplir (o si se quiere, son obedecidas) muy rara vez.

Solo con instituciones formales estables y que se cumplan regularmente podrían esperarse dos efectos centrales para la calidad de las políticas públicas: 1. que las expectativas de los actores entre sí sean también estables, y 2. que se produzcan cambios graduales, sea en los objetivos de una institución o bien en la institución como un todo. Este primer tipo de condiciones prevalece normalmente en los países capitalistas avanzados, y puede explorarse tanto desde una óptica de equilibrios puntuados por coyunturas críticas como desde una perspectiva de cambios graduales acumulativos (Pierson 2004; Thelen y Mahoney 2010). Las otras situaciones, y en particular el extremo de las instituciones débiles (cambios bruscos en las reglas formales y cumplimiento errático), describirían bastante bien los patrones de evolución política de una buena parte de los países latinoamericanos, en particular Venezuela, Colombia, Ecuador, Perú y Bolivia (Cameron 2018), y tendrían que modificarse los instrumentos teóricos estándar para la explicación del cambio político institucional.

Si en el enfoque PCP la estabilidad institucional es un resultado deseable -y en cierto sentido una marca normativa-, en la teoría de la variación institucional es posible identificar patrones de estabilidad e inestabilidad que deben ser explicados. ${ }^{14}$ En efecto, la duración de una institución formal puede ser el resultado de las estrategias (de uno o más actores poderosos) para estabilizar las expectativas de conducta que prevén algún tipo de retaliación si sus acciones no se ajustan a las reglas formales, pero también puede deberse a una estrategia de descuido; esto es, los intereses de los actores podrían estar dirigidos a mantener una institución formal que, se sabe, en la práctica no será

13. Los autores entienden a la estabilidad como la duración de las instituciones formales más allá de las condiciones iniciales en que fueron adoptadas, y al cumplimiento como el grado en que las reglas escritas son obedecidas en la práctica.

14. En este sentido, esta última perspectiva está más cercana al programa de investigación inicial del institucionalismo comparativo. 
obedecida. ${ }^{15}$ Finalmente, el patrón de inestabilidad y débil cumplimiento de las reglas formales deja de mirase como un efecto indeseable de la acción de actores poco cooperativos, y pasa a entenderse como producto de estrategias de actores que aumentan (o defienden) el control sobre varios tipos de recursos de poder precisamente porque las reglas existen, pero no se cumplen, o porque se espera que cambien por algún procedimiento abreviado.

También es posible que las variaciones en estabilidad y duración se deban a factores que los actores no controlan. Los diseñadores de las instituciones formales no necesariamente son iguales a los decisores políticos en un momento dado. El Estado ${ }^{16}$ podría carecer de las capacidades burocráticas y de penetración en el territorio y las sociedades bajo su dominio formal como para hacer cumplir las decisiones de las élites estatales; su poder infraestructural sería insuficiente (Soiffer y von Hau 2008; Altman 2012; Luna 2014; Andrade 2015). La presencia de fuertes y múltiples desigualdades entrecruzadas conspiraría contra el cumplimiento de las instituciones formales. La existencia de este amplio rango de factores causales puede, sin embargo, manejarse analíticamente mediante la exploración sistemática de las historias de surgimiento y modificación de las instituciones formales empíricamente existentes en un país (o sector) dado.

Adicionalmente, el método comparativo permite al enfoque de la fortaleza institucional (en adelante, EFI) rastrear la variabilidad institucional formal a través de varios períodos históricos, y precisar el ritmo de los cambios institucionales. Finalmente, el EFI da lugar a una intuición central para el análisis de los países en desarrollo: si bien las instituciones políticas formales son débiles, en tanto que reglas en el papel tienen el potencial de ser obligatorias. Esta última característica se respalda en tres condiciones.

En primer lugar, las constituciones, los tratados, las leyes y los reglamentos ${ }^{17}$ proveen una respuesta a las expectativas de los actores económicos.

15. Esta perspectiva tiene un gran poder interpretativo para casos como el de las sucesivas reformas judiciales desde 1979 para acá. Formalmente, ninguna de las tres constituciones vigentes durante ese período sometían al poder judicial o al ejecutivo y, además, en ningún momento el poder judicial logró constituirse en un poder autónomo (Basabe 2012).

16. El Estado es entendido en esta perspectiva como algo más que la sumatoria de las instituciones políticas formales.

17. Esto es, las instituciones políticas que los actores políticos poderosos han escrito según la tradición de derecho imperante en ese país en concreto. 
Por ejemplo, las regulaciones del mercado laboral en toda América Latina establecen la existencia de tres grandes grupos de actores - gobierno, representes de organizaciones del sector privado de la economía (empresarios) y trabajadores sindicalizados (organizaciones sindicales)-, señalan en qué condiciones deben actuar entre sí, y atribuyen al gobierno el poder decisivo (Schneider 2004 y 2013). Los participantes en el juego pueden esperar al menos dos posibles resultados: que se cumplan las reglas escritas, y que esa aplicación tenga en consideración los recursos de poder que cada actor puede movilizar. En principio, el gobierno de cualquier país latinoamericano, en la medida que posea autoridad política nacional, podría tratar de imponer su voluntad con mayor facilidad que las organizaciones de empresarios y trabajadores. A diferencia del enfoque PCP, el EFI no da por supuesta la autoridad estatal, pero considera que los otros actores políticos no pueden dar por descontado -todo el tiempo y en cada encuentro- que el gobierno se abstendrá de hacer cumplir la literalidad de la ley.

El EFI crea un rango de marcos analíticos enfocados sensibles a las condiciones empíricas (internacionales, geográficas, temporales) del suelo en el que ocurren las relaciones de autoridad entre gobiernos y actores sociales organizados. Al hacerlo, traza una frontera entre las instituciones formales y las informales, y ahí se detiene. El campo del estudio de las instituciones que se hallan más allá de la línea de la formalidad está excluido de esta vertiente de la economía política. Esta última pretensión teórica es la que asume el enfoque de los acuerdos/fundamentos políticos básicos (en adelante, EAF).

\section{Distribución del poder organizacional y transformación institucional}

El EAF parte de una afirmación institucionalista radical. En países en desarrollo, el suelo en el cual se crean las decisiones políticas es el resultado de distribuciones de recursos de poder que controlan los actores -y que no están escritas en los códigos legales imperantes en esos países-y las instituciones políticas formales. De hecho, esa superficie es dinámica ("inestable" y "débil", en la terminología del PCP y el EFI) porque la adopción de estas últimas reglas es un intento de la coalición política gobernante por cambiar la 
distribución de poder político existente. ${ }^{18}$ El proceso de estabilización de los fundamentos políticos de un país depende, entonces, de la alineación entre la distribución de recursos de poder que consagran las reglas formales y la capacidad de los actores para mantener el control de esos recursos. Para la perspectiva, la negociación entre actores poderosos no culmina con la adopción de una institución formal, es su inicio; el proceso se extiende hacia la aplicación selectiva de las reglas formales. Esta dinámica es en cierta medida independiente del tipo de régimen (autoritario o democrático) formalmente adoptado por las élites gobernantes de un país; lo que cuenta es el tipo de coalición ${ }^{19}$ gobernante y si las reglas que estas adoptan para ejercer el gobierno son compatibles o no con la distribución de poder entre los actores.

Para el EAF, por defecto, la organización básica del poder en los países en desarrollo ${ }^{20}$ está estructurada en redes de patronazgo o clientelares. En cualquier momento dado, múltiples redes de patronazgo compiten entre sí por mantener e incrementar los recursos de poder que controlan. El control del aparato estatal es la frontera que marca quiénes forman parte de una coalición gobernante. ${ }^{21}$ Por lo tanto, y desde el punto de vista de la distribución de recursos de poder, la coalición gobernante despliega actividades horizontalmente en relación con aquellas organizaciones excluidas de la coalición gubernamental, y verticalmente hacia las facciones que son miembros de la coalición. En el primer caso, la acción está orientada a mantener la exclusión

\section{Usualmente a su favor.}

19. Leftwich y Hogg definen a las coaliciones como "individuos, grupos u organizaciones que se alían para alcanzar metas económicas y políticas que no serían capaces de alcanzar por sí solos" $(2007,8)$. Las coaliciones difieren de las organizaciones en cuanto a su duración, y en que los miembros de una coalición retienen sus identidades políticas individuales.

20. El debate en el que se crea este enfoque se ha dado en Europa, Reino Unido y Australia, principalmente, en torno a las políticas de cooperación internacional para el desarrollo orientadas hacia Asia y África. No debería sorprender que algunos de los participantes más destacados de esta comunidad académica sean economistas del desarrollo de origen asiático (Kahn 2010; Sen 2015; Ang 2016a y 2016b) y africano (Kahn 2018). La presencia de latinoamericanos es, en cambio, menor, aunque puede argüirse que existe una sólida tradición en los estudios latinoamericanos del desarrollo que avanzó esa relación de causalidad desde el estructuralismo y el marxismo (Thorp 1998; Kay 1991).

21. Kahn (2018) admite la posibilidad que organizaciones poderosas que no forman parte de la coalición de gobierno puedan iniciar cambios de las instituciones formales (por ejemplo, presentando iniciativas legislativas, o mediante el cabildeo con decisores políticos); sin embargo, en la situación estándar, la iniciativa recae en la coalición gobernante. 
o debilitar el poder organizacional de las facciones excluidas; en el segundo, a consolidar la coalición gobernante mediante el reparto de beneficios materiales y simbólicos a sus miembros. La variación en estas dos dimensiones determina el horizonte temporal de la coalición gobernante y sus capacidades para implementar las reformas institucionales formales y las políticas económicas y sociales que haya adoptado (Kahn 2010).

Metodológicamente, el EAF centra su análisis en el poder organizacional de las facciones excluidas/incluidas en una coalición gubernamental. La capacidad de un actor (organización) para interactuar con la coalición gobernante depende de tres factores: en primer lugar, de su habilidad para movilizar los recursos de $\operatorname{poder}^{22}$ que controla vis-à-vis la coalición de gobierno; segundo, del tipo de recursos (de propiedad, normativos, de coerción y de la fuerza laboral, principalmente) que los actores controlan; y, tercero, de la relevancia de esos recursos para el intercambio con la coalición de gobierno en un momento dado (por ejemplo, ante una iniciativa gubernamental para adoptar una política económica o social).

La capacidad de las organizaciones para mantener los recursos que controlan y movilizarlos en la situación relevante constituye su poder organizacional. El empleo de este poder involucra costos de movilización y aplicación. Empíricamente, la identificación de esos costos depende del tipo de posición que ocupen los actores con respecto a la coalición gobernante; esto es, de la capacidad del liderazgo de las facciones para identificar cuándo y cómo van a poner en marcha los recursos que controlan para recompensar a los miembros de la organización y castigar a los que pertenecen a la coalición gobernante (u otras organizaciones rivales), a través de redes formales o informales (Kahn 2018). Dado que los conflictos entre coaliciones ocurren de manera repetida, los costos en los que incurren las organizaciones no pueden comprometer su fortaleza de cara al siguiente enfrentamiento. El análisis adquiere, por lo tanto, un fuerte sesgo histórico, toda vez que la descripción de la distribución del poder organizacional en una sociedad se obtiene mediante el examen histórico de las distintas coaliciones de poder y sus intentos por

22. Korpi (1985) define a los recursos de poder como los atributos (capacidades o medios) de los actores que les permiten recompensar o castigar a otros actores. Los atributos se convierten en recursos solo cuando los actores interactúan. La movilización de esos recursos es el ejercicio del poder. 
estabilizar una cierta configuración general de redes clientelares, así como de las organizaciones que se han movilizado y han perdido o ganado a lo largo del tiempo (Kahn 2018).

En el EAF, la pregunta ha dejado de ser por la calidad (fortaleza) de las instituciones o su diseño (si favorecen o no interacciones cooperativas), sino si las relaciones entre actores poderosos permiten la emergencia de ciertas instituciones formales, si estas sobreviven a través de varios gobiernos y formas de régimen, y cómo son implementadas dentro de los límites impuestos por la distribución de poder en la sociedad. En la medida que la interacción entre intentos por imponer reglas formales involucra costos de movilización e implementación para la coalición de gobierno que inicia el cambio, es de esperarse que esos costos se manifiesten a la manera de violación de las reglas, inestabilidad institucional (cambios bruscos en la literalidad de los cuerpos legales) y corrupción.

La fluidez de las interacciones entre las reglas formales y las organizaciones poderosas (formales e informales) es el estado esperado. El equilibrio entre actores solo se obtendrá cuando las instituciones políticas formales sean compatibles con la distribución de poder entre las redes clientelares de una sociedad; si esto ocurre, entonces emerge un acuerdo/fundamento político que tiende a sostenerse en el tiempo. Lo que es más importante: aun si se obtiene algún equilibrio, es solamente temporal, porque las organizaciones cambian en la medida que su capacidad para mantener su posición se fortalece o debilita, así como por el surgimiento de nuevos grupos sociales que pueden ser incorporados o no a la coalición de gobierno. Sin embargo, una vez que se ha estabilizado un cierto equilibrio -que ha emergido un acuerdo/fundamento político-, la introducción de nuevas instituciones políticas formales generará resistencias que explican el rendimiento de estas innovaciones. De esta manera, el EAF puede explicar la naturaleza conflictiva del cambio institucional (Kahn 2018).

En el marco de los acuerdos/fundamentos políticos, no se asume la bondad a priori de la inclusividad de una institución política en particular. ${ }^{23} \mathrm{En}$

23. Recuérdese que el enfoque del proceso político pone un premio en las instituciones inclusivas. Coincide en este aspecto con la afirmación de Acemoglu y Robinson (2012) de que, a medida que las instituciones políticas se vuelvan más incluyentes, tenderán a producir mejores instituciones económicas en relación con la distribución de los resultados del crecimiento económico. 
efecto, en las condiciones imperantes en los países en desarrollo, las organizaciones que controlan recursos económicos (principalmente, la propiedad y la capacidad para establecer el precio de la fuerza laboral) bien podrían no estar interesadas en incrementar la competencia en el mercado, peor aún en remover las regulaciones que les permiten capturar rentas; por otro lado, los sectores populares -o incluso la clase media- carecen de poder organizacional. Así, la introducción de instituciones políticas que favorezcan la participación de los actores poderosos en la toma de decisiones tendería a fortalecer el poder político de las organizaciones económicamente poderosas $\mathrm{y}$, por lo tanto, a dificultar la distribución de los beneficios del crecimiento. De igual manera, el EAF pone en duda el optimismo del EFI sobre las expectativas de cumplimiento de las leyes: una coalición de gobierno bien podría ser consistente en la aplicación de todo tipo de regulaciones para con las facciones excluidas, pero no someterse a sí misma al imperio de la ley, y actuar muy selectivamente con relación a las facciones subordinadas de la propia coalición (Andrade 2015).

En síntesis, el EAF hace posible que el análisis económico político (del desarrollo, en particular) expanda su campo mediante la incorporación de análisis históricos sobre la distribución de poder en una sociedad dada, y de investigaciones sociológicas y antropológicas sobre las trayectorias de formación de organizaciones con capacidad para acumular y movilizar el control de recursos de poder, así como del lugar que ocupan las organizaciones estatales en esa sociedad. El grado de complejidad que emerge en esta versión de la economía política parecería a primera vista intratable; sin embargo, para propósitos analíticos es posible delimitar esa complejidad a partir del concepto de acuerdos/fundamentos políticos básicos -estableciendo configuraciones relativamente coherentes de instituciones formales e informales que se han mostrado sostenibles durante períodos relativamente largos- y marcar cuándo la intensificación de los conflictos en torno a las instituciones formales da paso a transformaciones rápidas de estas últimas. ${ }^{24}$ Adicionalmente, al relacionar esos períodos de estabilidad y cambio brusco

24. Los cambios bruscos se conciben desde la perspectiva como resultado de actividades disruptivas de facciones poderosas excluidas o bien de facciones subordinadas que han ganado acceso a nuevos recursos de poder, cuando esta nueva posición no es compatible con las instituciones adoptadas por las facciones dominantes de una coalición de gobierno. 
con indicadores de crecimiento económico y exclusión/inclusión social, el enfoque permite explicar la trayectoria típica de los países en desarrollo, caracterizada por períodos de rápido crecimiento económico seguidos de fases de estancamiento o incluso colapso económico (Sen 2015).

\section{Implicaciones para la investigación futura}

La discusión precedente indica que el debate académico en lo que va del siglo ha inaugurado un nuevo campo de investigación para América Latina: el de la política de las políticas. El examen anterior ha mostrado que se trata de un campo en disputa entre varios marcos teóricos enfocados, cuyos supuestos, métodos y énfasis explicativos difieren entre sí. Sin embargo, también es posible afirmar que, a pesar de esas diferencias, los enfoques revisados comparten al menos tres elementos en común. En primer lugar, es inadecuada la caracterización de la política latinoamericana en términos de las instituciones políticas formales de una democracia abstracta que asuma como estándar a las democracias de los países capitalistas avanzados. En América Latina se puede comprobar la coexistencia entre una formalidad democrática que importa, pues al regular la competencia política por el control del Estado tiene un peso específico, y simultáneamente no importa, porque las instituciones políticas, a pesar de guardar coherencia entre sí (formar un régimen), varían en su capacidad para enmarcar el comportamiento político de los actores. Segundo, el diseño o incluso el contenido de una institución política formal es menos importante que los mecanismos políticos por los cuales esa institución es sostenida en el tiempo y adquiere fuerza obligatoria, toda vez que estos dos últimos resultados dependen de factores endógenos al conjunto de procesos sociales que las instituciones formales pretenden regular. A la inversa, que las instituciones formales sirvan de reglas de juego, estabilizando las expectativas de los actores y favoreciendo que estos inviertan en el mantenimiento de esas reglas, no depende de cuán democráticas o autoritarias sean esas reglas o su ejercicio, sino de si pueden o no de hecho ejercerse. Estos dos elementos han movido el objeto de análisis al estudio de las relaciones de poder entre los actores organizados. 
Finalmente, es claro que si las relaciones de poder entre organizaciones son el nuevo campo en el que se realiza la exploración, el esfuerzo de investigación no puede seguir empleando una concepción estrecha y sustantiva del poder. Los tres marcos analíticos examinados retienen una concepción común de la acción política como una actividad estratégica, orientada a fines, pero su grado de individualismo metodológico varía; la agencia de los actores solo es posible cuando se cumple el requisito (lógica y empíricamente previo) del acceso a recursos de poder. Los actores involucrados en la creación de políticas son, en definitiva, organizaciones, aun cuando líderes individuales puedan movilizar recursos normativos y de otro tipo para formar coaliciones. Los líderes de organizaciones y coaliciones ciertamente se pintan como gerentes de recursos de poder, pero esa gerencia solo puede ejercerse en un contexto estructurado por la presencia de otras organizaciones y reglas formalizadas. Finalmente, estas últimas son concebidas como rastros (o si se prefiere, depósitos) de luchas anteriores por estabilizar las relaciones entre actores.

Si bien difieren las explicaciones de la fortaleza de las instituciones formales que los tres enfoques ofrecen sobre la variación intertemporal y longitudinal, también es cierto que hay algunas condiciones causales en las que coinciden. En primer lugar, los tres enfoques registran la variación institucional como un problema de investigación, y todos ellos coinciden en que las condiciones locales imperantes (y no la adopción de mejores prácticas o mejores diseños) son los factores fundamentales en la manera en que las instituciones políticas efectivamente funcionan en un país en particular. La implicación directa para investigaciones futuras es clara: el examen de trasplantes institucionales desde la perspectiva del diseño es un camino poco fructífero; en su lugar emergen preguntas por el tipo de coaliciones que impulsan reformas institucionales, y por los potenciales (o actuales) efectos de la introducción de una reforma institucional en relación con la distribución de recursos de poder entre los actores poderosos presentes en una sociedad. En segundo lugar, tanto el EFI como el EAF iluminan los costos de transición institucional; esto es, dado que una reforma institucional formal involucra la movilización de recursos de poder por parte de quienes la inician, cabe esperar que actores individuales y organizaciones también movilicen sus recursos para resistir la aplicación de las nuevas instituciones. Acá emergen preguntas no solo sobre el tipo de recursos puestos en juego en condiciones 
de conflicto abierto, sino también sobre las estrategias de los actores que llevan al conflicto abierto y no a otras formas de ejercicio de poder (negociación o explotación). Finalmente, las tres teorías resaltan la fuerza causal de la dependencia de trayectoria en el cambio institucional; es decir, las luchas en torno al cambio de instituciones políticas formales generan experiencias de aprendizaje en los actores, y esas experiencias definen no solo la estabilidad de sus expectativas, sino también -y lo más importante- los intereses mismos de los actores y el rango de estrategias que están dispuestos a emplear. Este es un descubrimiento fundamental para la comparación entre distintos modos de evolución institucional (rápido o gradual), la determinación de si y qué constituye una coyuntura crítica, y la comparación entre coyunturas críticas.

\section{Referencias}

Acemoglu, Daron, y Robinson James. 2012. Why Nations Fail: The Origins of Power, Prosperity, and Poverty. Nueva York: Crown.

Altman, David. 2005. "La institucionalización de la ciencia política en Chile y América Latina: una mirada desde el Sur". Revista de Ciencia Política 25 (1): 3-15. http://dx.doi.org/ 10.4067/S0718-090X2005000100001

---.2012. "Where is Knowledge Generated? On the Productivity and Impact of Political Science Departments in Latin America". European Political Science 11 (1): 71-87. https:// bit.ly/2NAqWGM

Andrade, Pablo. 2008. Democracia y cambio político en el Ecuador: liberalismo, política de la cultura y reforma institucional. Quito: Universidad Andina Simón Bolívar, Sede Ecuador / Corporación Editora Nacional.

---. 2015. Política de industrialización selectiva y nuevo modelo de desarrollo. Quito: Universidad Andina Simón Bolívar, Sede Ecuador / Corporación Editora Nacional.

Ang, Yuen Yuen. 2016a. "Beyond Weber: Conceptualizing an Alternative Ideal Type of Bureaucracy in Developing Contexts". Regulation \& Governance 11 (3): 282-298. https:// doi.org/10.1111/rego.12123

---. 2016b. How China Escaped the Poverty Trap. Ithaca: Cornell University Press.

Basabe, Santiago. 2012. "Judges without Robes and Judicial Voting in Contexts of Institutional Instability: The Case of Ecuador's Constitutional Court, 1999-2007”. Journal of Latin American Studies 44 (1): 127-161. https://www.jstor.org/stable/41349722

Cameron, Maxwell A. 2018. "The Survival of Defective Democracies". University of British Columbia. Accedido 20 de mayo de 2019. https://bit.ly/37q4BDp 
Carey, John M. 2000. "Parchment, Equilibria, and Institutions". Comparative Political Studies 33 (6/7): 735-761. https://doi.org/10.1177/001041400003300603

Evans, Peter, y Barbara Stallings. 2016. "Development Studies: Enduring Debates and Possible Trajectories". Studies in Comparative International Development 51 (1): 1-31. https:// doi.org/10.1007/s12116-016-9223-9

Greene, Kenneth. 2009. Why Dominant Parties Lose: Mexico's Democratization in Comparative Perspective. Nueva York: Cambridge University Press.

Jones, Mark P. 2017. How Much Has the Game Changed? Revisiting Policymaking in Latin America a Decade Later. Washington D. C.: IDB.

Kahn, Mushtaq H. 2010. "Political Settlements and the Governance of Growth-Enhancing Institutions". SOAS University of London. Accedido 11 de septiembre de 2019. http:// eprints.soas.ac.uk/id/eprint/9968

---. 2018. "Political Settlements and the Analysis of Institutions". African Affairs 117 (469): 636-655. https://doi.org/10.1093/afraf/adx044

Kay, Cristóbal. 1991. “Teorías latinoamericanas del desarrollo”. Nueva Sociedad 113: 101-113. https://bit.ly/2NDcEVT

Korpi, Walter. 1985. "Power Resources Approach vs. Action and Conflict: On Causal and Intentional Explanations in the Study of Power". Sociological Theory 3 (2): 31-45. https:// doi.org/10.2307/202223

Leftwich, Adrian, y Steve Hogg. 2007. "The Case for Leadership and the Primacy of Politics in Building Effective States, Institutions and Governance for Sustainable Growth and Social Development". Wageningen University \& Research. Accedido 2 de septiembre de 2019. https://bit.ly/2qRzuzQ

Levitsky, Steven, y María Victoria Murillo. 2009. "Variation in Institutional Strength". Annual Review of Political Science 12 (1): 115-133. https://doi.org/10.1146/annurev.polisci.11.091106.121756

Luna, Juan Pablo. 2014. Segmented Representation: Political Party Strategies in Unequal Democracies. Oxford: Oxford University Press.

Mazzucato, Mariana. 2018. The Value of Everything: Making and Taking in the Global Economy. Nueva York: Hachette Book Group.

Pierson, Paul. 2004. Politics in Time: History, Institutions, and Social Analysis. Princeton: Princeton University Press.

Polga-Hecimovich, John, y Francisco Sánchez. 2018. "The Tools of Institutional Change under Post-Neoliberalism: Rafael Correa's Ecuador". Journal of Latin American Studies 51 (2): 379-408. https://doi.org/10.1017/S0022216X1800072X

Rodrik, Dani. 2007. One Economics, Many Recipes: Globalization, Institutions, and Economic Growth. Princeton: Princeton University Press.

Rueschmeyer, Dietrich. 2009. Usable Theory: Analytic Tools for Social and Political Research. Princeton: Princeton University Press. 


\section{Pablo Andrade Andrade}

Schneider, Ben-Ross. 2004. Business Politics and the State in Twentieth-Century Latin America. Cambridge: Cambridge University Press.

---. 2013. Hierarchical Capitalism in Latin America. Business, Labor, and the Challenges of Equitable Development. Nueva York: Cambridge University Press.

Sen, Kunal. 2015. "The Political Determinants of Economic Growth: An Analytical Review". En The Politics of Inclusive Development, editado por Sam Hickey, Kunal Sen y Badru Bukenya, 35-59. Oxford: Oxford University Press.

Silva, Eduardo. 2009. Challenging Neoliberalism in Latin America. Nueva York: Cambridge University Press.

---, y Federico Rossi. 2018. From Resistance to Neoliberalism to the Second Wave of Incorporation: Comparative Perspectives on Reshaping the Political Arena. Pittsburgh: University of Pittsburgh Press.

Soifer, Hillel, y Matthias vom Hau. 2008. "Unpacking the Strength of the State: The Utility of State Infraestructural Power". Studies in Comparative International Development, 43 (3-4): 219-230. 10.1007/s12116-008-9030-z

Spiller, Pablo, Eduardo Stein y Mariano Tommasi. 2006. "Political Institutions, Policymaking, and Policy: An Introduction". En Policymaking in Latin America. How Politics Shapes Policies, editado por Pablo Spiller, Mariano Tommasi, Ernesto Stein y Carlos Scartascini, 1-27. Washington D. C.: IBD.

Thelen, Kathleen, y James Mahoney. 2010. “A Theory of Gradual Institutional Change”. En Explaining Institutional Change: Ambiguity, Agency and Power, editado por James Mahoney y Kathleen Thelen, 1-36. Nueva York: Cambridge University Press.

Thorp, Rosemary. 1998. Progress, Poverty, and Exclusion. An Economic History of Latin America in the $20^{\text {th }}$ Century. Washington D. C.: IDB.

Zibblat, Daniel. 2006. “How Did Europe Democratize?”. World Politics 58 (2): 311-338. https://doi.org/10.1353/wp.2006.0028 